\title{
Cooperation in An Overlapping Generations Experiment ${ }^{*}$
}

\author{
Theo Offerman, Jan Potters and Harrie Verbon
}

November 1998

\section{Abstract}

Recent theoretical work shows that folk theorems can be developed for infinite overlapping generations games. Cooperation in such games can be sustained as a Nash equilibrium. Besides the efficient cooperative equilibrium there is also the inefficient non-cooperative equilibrium. This paper investigates experimentally whether cooperation actually occurs in a simple overlapping generations game. Subjects both play the game and formulate full strategies. In our base-line treatment subjects fail to exploit the intertemporal structure of the game. When we provide subjects with a recommendation to play a (subgame perfect) grim trigger strategy, a substantial part of the population develops trigger-like strategies. However, a remaining group of unconditional defectors prevents the cooperative outcome to evolve.

JEL codes: C72, C92, D90.

Key-words: overlapping generations, cooperation, trigger strategies, experiments

Theo Offerman

University of Amsterdam

Department of Economics /CREED

Roetersstraat 11

1018 WB Amsterdam

Netherlands

e-mail: theoo@fee.uva.nl
Jan Potters and Harrie A.A.Verbon

Tilburg University

CentER/Department of Economics

Warandelaan 2

5000 LE Tilburg

Netherlands

j.j.m.potters@kub.nl,h.a.a.verbon@kub.nl

* We gratefully acknowledge financial support from the Netherlands Organization of Scientific Research (NWO). The research by Jan Potters is made possible by a fellowship of the Royal Netherlands Academy of Arts and Sciences. We thank Henk OpdenBrouw for programming the experiment. 


\section{Introduction}

The overlapping generations model, first introduced by Samuelson (1958), has become a standard tool in economics. It has been used to study a wide variety of issues, such as the sustainability of old-age pension schemes (Hammond, 1975), the time-consistency of optimal tax policies (Kotlikoff et al., 1988), the relation between growth and environment (John and Pecchenino, 1994), the conditions for the optimal provision of club-goods (Sandler, 1982), the relation between regulators and firms (Salant, 1995), and the interaction between old and young members of a political party (Alesina and Spear, 1987). In many of these applications, a central question is whether and under what conditions some form of efficient or cooperative outcome can be sustained as an equilibrium. Often it is found that, under certain conditions, this is indeed the case. For example, Hammond shows that an efficient pay-as-you-go pension scheme can be supported as an equilibrium outcome if the players employ trigger strategies. ${ }^{1}$

In all of these studies cooperation is one of the equilibrium outcomes, but not the only one. As in infinitely repeated games, typically, there are many equilibria in infinite overlapping generations games. Sometimes additional criteria are advanced to argue that a particular equilibrium is more reasonable. For example, Hammond (1975) suggests that the players will realize that the cooperative equilibrium is in their own interest and that it is reasonable to assume that they will coordinate on this equilibrium. Usually, however, authors seem content in having established that a particular cooperative or efficient outcome is an equilibrium. Whether that particular equilibrium is more reasonable or more likely to be played is not addressed. Ultimately, of course, this is an empirical matter (Lucas, 1986).

In the present paper we 'put the theory to the test'. We let subjects play a simple overlapping generations game. The structure of the game is identical to the Pension Game studied by Hammond (1975). It is the most simple overlapping generations game that is not strategically trivial. The game is played by an infinite sequence of players, $\mathrm{P}_{1}, \mathrm{P}_{2}, \mathrm{P}_{3}, \ldots$ The first player $\mathrm{P}_{1}$ does not make a choice. Each subsequent player makes one choice only from the set $\{\mathrm{A}, \mathrm{B}\}$. If the second player $\mathrm{P}_{2}$ chooses $A(B), P_{1}$ receives 50 (30). Each subsequent player $\mathrm{P}_{t}$ 's payoff is determined by his own choice and by the choice of the next player $P_{t+1}$, his successor $(t \geq 2)$. Table 1 shows the payoff scheme. This payoff scheme is common knowledge.

\footnotetext{
${ }^{1}$ The possibility of cooperation in overlapping generations has been studied and established more generally. Folk theorems may arise, similar to the ones for 'genuine' infinitely repeated games (Cremer, 1986, Salant, 1991, Smith, 1992).
} 
Table 1

Payoff scheme

\begin{tabular}{|c|c|c|c|}
\hline \multicolumn{2}{|c|}{ payoff to player $\mathrm{P}_{t}$} & \multicolumn{2}{|c|}{ choice $P_{t+1}$} \\
\hline & & A & $\mathrm{B}$ \\
\hline & A & 50 & 15 \\
\hline $\cos ^{2}$ & $\mathrm{~B}$ & 70 & 30 \\
\hline
\end{tabular}

In this game there are two focal outcomes: one non-cooperative and one cooperative. If all players choose B, then all will earn 30 . We call this the non-cooperative outcome. Informally, this outcome is an equilibrium according to the following argument. If player $\mathrm{P}_{\mathrm{t}}$ believes that the choice by player $\mathrm{P}_{\mathrm{t}+1}$ will be independent of his own choice, then clearly it is in his best interest to choose $\mathrm{B}$. Whatever the choice of $\mathrm{P}_{\mathrm{t}+1}(\mathrm{~A}$ or $\mathrm{B}$ ), if it does not depend on the choice of $P_{t}$, then it is a best reply for $P_{t}$ to play $B$. This argument is similar to the dominance argument that leads to non-cooperation in the prisoner's dilemma.

If all players choose to play A, however, then all will receive a payoff of 50 . We can call this the cooperative outcome. It can be supported by some form of trigger strategy. Suppose that player $\mathrm{P}_{\mathrm{t}}$ believes that player $\mathrm{P}_{\mathrm{t}+1}$ will play $\mathrm{A}$ if and only if all previous players have played $\mathrm{A}$, then it will be in his interest to play $\mathrm{A}$ if and only if all previous players have played A. Therefore, this 'grim trigger' strategy is self-enforcing. The cooperative outcome can be supported as a subgame perfect equilibrium if each player is informed about the choices of all previous players, and if there is an infinite sequence of players (cf. Bhaskar, 1994).

In our base-line treatment we investigate whether subjects spontaneously devise trigger strategies supporting the cooperative outcome. Subjects both make a choice and formulate a strategy to play the game. The latter information gives us more direct and detailed evidence on the strategies considered by our subjects.

In our recommendation treatment we provide subjects with a recommendation supporting the cooperative equilibrium. This recommendation was suggested by one of the subjects in the base-line treatment. It amounts to the grim trigger strategy. This strategy has the advantage of being both simple (contrary to, e.g., 'defector-detector') and subgame perfect (contrary to, e.g., 'tit-for-tat'). ${ }^{2}$ Previous experimental work shows that recom

\footnotetext{
${ }^{2}$ Consider the (tit-for-tat) strategy profile in which each player plays A if and only if her predecessor played A. Suppose player $P_{t-1}$ played A. Then player $P_{t}$ is supposed to play A as well. Given that player
} 
mendations affect subjects' play in some circumstances. Van Huyck, Gilette and Batallio (1992) find the result that subjects tend to follow equilibrium recommendations as long as they do not conflict with payoff-dominance or symmetry. The results by Brandts and McLeod (1995) are mixed in this respect. In some of their games recommendations matter for subjects' play. However, they also construct a game where the perfect equilibrium does not coincide with the Pareto-efficient equilibrium. In that game subjects do not tend to follow the recommended efficient equilibrium strategy.

Ours is not the first experimental study of overlapping generations. For example, Cadsby and Frank (1990) study Ricardian equivalence in an overlapping generations experiment. Lim, Prescott, and Sunder (1994), and Marimon and Sunder (1993) study expectations formation in an experiment in which the value of money savings depends on future prices as determined by the behavior of future generations. These experiments have a different focus than ours, and are much more complex than our simple 'pension game'. A paper with a focus similar to the present one is van der Heijden et al. (1998). They study the occurrence of cooperative transfers in a finite overlapping generations game. Strictly speaking, however, in their experiment cooperation is not an equilibrium. Furthermore, in all of these studies the subjects play the game repeatedly which may introduce other reasons for cooperation than those purely based on the dynamic structure of the overlapping generations game. It is the latter that is the focus of the present study. Moreover, by using the strategy method (alongside simple play of the game) we can see whether subjects actually use trigger-like strategies to exploit the dynamic structure of the game.

\section{Experimental design and procedure}

The experiment was conducted with the help of the computer. ${ }^{3}$ The instructions are distributed to subjects and read aloud. The Appendix contains a full transcription. We use neutral terminology when explaining the game. For example, we avoid words like 'cooperating'. First we explain the base-line treatment. Then we will focus on the recommendation treatment.

$\mathrm{P}_{\mathrm{t}+1}$ plays tit-for-tat, it is in $\mathrm{P}_{\mathrm{t}}$ 's interest to indeed play A. Hence, tit-for-tat is a Nash equilibrium. It is not subgame-perfect, however. Suppose that player $\mathrm{P}_{\mathrm{t}-1}$ plays the (off-equilibrium) action $\mathrm{B}$. Then player $P_{t}$ is supposed to play $B$. However, given that player $P_{t+1}$ plays tit-for-tat it is clearly in $P_{t}$ 's interest to play A instead.

\footnotetext{
${ }^{3}$ We are grateful to Abbink and Sadrieh for letting us use their RatImage library.
} 
In the experiment, of course, it is impossible to have an infinite sequence of players, and we use the following stopping rule. After player $\mathrm{P}_{2}$ has made her choice, there is $90 \%$ probability that an additional player, $\mathrm{P}_{3}$, is added to the sequence, and a $10 \%$ probability that player $\mathrm{P}_{2}$ is the last player. In the latter case the game ends, and the final player ( $\mathrm{P}_{2}$ in this case) earns a fixed payoff of 40 guilders. In the former case the game continues and player $\mathrm{P}_{3}$ makes a choice. Table 1 shows the payoffs in guilders for subjects that are not at the beginning or the end of the sequence. ${ }^{4}$ Then a 90-10 lottery again determines whether or not an additional player, $\mathrm{P}_{4}$, is added to the sequence. This procedure is repeated until the game ends. ${ }^{5}$ As we explain to our subjects, we carried out this lottery procedure before the start of the experiment. Subjects are randomly allocated a position in the sequence. A subject makes at most one choice in the sequence. We invited more people to the experiment than the number determined by the lottery. Therefore, some subjects do not make a choice at all, because the sequence is closed before it is their turn to choose. Subjects who do not make a choice earn a fixed payoff of 40 guilders.

When the basic structure of the game has been explained to the subjects, they have ample time to practice. In the practicing phase subjects make choices for all participants in the row (to avoid any guidance about what to choose). When a subject enters a choice, the computer determines the corresponding payoffs and whether an additional player is added to the sequence, for which the subject then again enters a choice. In some practicing rounds the subjects are requested to enter the corresponding payoffs themselves, and then computer reports back whether the answer is correct or not.

After the practicing phase subjects are explained that they also have to write a strategy for this game. A strategy for this game requires a player to make a decision for any possible position in the sequence, and for any possible history of choices by earlier players in the row. To help subjects formulate a strategy, we provide them with some forms containing 'strategy classes' (see appendix). The experiment is carried out twice, once with the formulated

\footnotetext{
${ }^{4}$ The structure of the payoffs is similar to a prisoner's dilemma but the values are to a large extent arbitrary. In comparison to other prisoner's dilemma experiments the values do not seem particularly conducive to either cooperation or non-cooperation though. The 'cooperation index' (Rapoport and Chammah, 1965) in our game is 0.36, as compared to, for example, 0.26 in Selten and Stoecker (1986) and 0.39 in van der Heijden et al. (1998).

${ }^{5}$ It is easily shown that also with this stopping rule, the cooperative outcome (always A) can be supported as a subgame perfect equilibrium.
} 
strategies and once in the natural time order. When making a choice in the natural time order, a subject has information about all choices of her predecessors.

Subjects formulate a strategy before they choose in the natural time order. The results of the strategy experiment are communicated only after the end of the natural time experiment to avoid learning about other subjects' strategies. In playing out the strategy experiment, subjects are put in the same row and place as in the baseline experiment. Before subjects are paid they fill out a post-experimental questionnaire. Finally, a subject's throw with a die determines whether the strategy experiment (outcome 1,2,3) or the natural time order experiment (outcome 4,5,6) is used for actual payment.

In the recommendation treatment subjects receive the following recommendation at the end of the instructions (translated from Dutch): ${ }^{6}$

'A participant of a previous experiment made a recommendation to participants of future experiments which amounted to: "I recommend the second participant to choose A; I recommend each subsequent participant to choose A if all previous participants in the row chose A and to choose B otherwise. If everybody sticks to this recommendation, then it is in each participant's interest to stick to it. A choice for A is then 'rewarded' with a choice for A by the subsequent participant, while a choice for B is 'punished' with a choice for B by the subsequent participant in the sequence. In this way the aggregate payoff of all participants will be as large as possible."'

This is the only difference between the recommendation treatment and the base-line treatment.

\section{Subjects}

Subjects are recruited at Tilburg University. A total of 77 subjects participate in the experiment: 39 in the base-line treatment and 38 in the recommendation treatment. In each treatment 4 independent sequences are formed with lengths of $8,8,12$ and 7 players. $^{7}$ The

\footnotetext{
${ }^{6}$ In the post-experimental questionnaire such a recommendation was actually made by some of the participants in the baseline treatment. We preferred to give a recommendation by one of the subjects rather than presenting it as our recommendation in order to avoid an experimenter's induced bias.

${ }^{7}$ Per session we ran two sequences (rows) at the same time. This procedure prevents subjects to anticipate the length of a sequence from the number of subjects in the room. For example, subjects in the sequence of 11 players were part of a group of 23 subjects. In the recommendation treatment, we had to finish the planned sequence of 12 after the eleventh player because some subjects failed to show up.
} 
remaining subjects do not play in the natural time order experiment, but they do formulate a strategy.

\section{Results}

\section{Basic analysis of choices and strategies}

Before we discuss the strategies formulated by our subjects, we analyze the choices made in the natural time order experiment. In the base-line treatment subjects appear not to succeed in exploiting the intertemporal structure of the game to enforce the cooperative outcome. Here, only $16.1 \%$ (5/31) cooperative choices are made. The recommendation offered in the recommendation treatment does stimulate cooperative play to some extent. In this treatment subjects make $36.7 \%$ (11/30) cooperative choices. The difference in cooperative play between the baseline treatment and the recommendation treatment is marginally significant (Mann-Whitney rank test: $\mathrm{m}=4, \mathrm{n}=4 ; \mathrm{p}=0.06)^{8}$

There is some indication that cooperation in the recommendation treatment is to some extent history dependent. Cooperative choices are followed by a cooperative choice in $60.0 \%$ of the cases, whereas a non-cooperative choice is followed by a cooperative choice in only $29.4 \%$ of the cases. In the base line treatment, on the other hand, no cooperative choice is followed by another cooperative choice (but here, of course, we have few cooperative choices in all). ${ }^{9}$

The finding that subjects' choices are affected by the recommendation is also reflected in results of the post-experimental questionnaire. When asked about their considerations in the natural time order experiment the subjects in the base line treatment expressed the greatest average agreement with the statement: "It is always best to choose B irrespective of the choices of previous participants", whereas subjects in the recommendation treatment agreed most with the statement: "Your own choice affects the choice that your successor will make".

\footnotetext{
${ }^{8}$ Average payoffs were 33.07 in the base line treatment (15.00 for A choices, 36.96 for B choices) and 40.00 in the recommendation treatment ( 36.00 for A choices, 42.50 for B choices).

${ }^{9}$ One may also wonder whether cooperative choices are typically made earlier or later in the sequence. To investigate this we compare the average rank numbers (place in the row) of subjects choosing cooperatively with those choosing noncooperatively. Aggregated over the two treatments the average rank number is 5.32 for a cooperative choice and 5.28 for a noncooperative choice. Hence, there does not seem to be a difference of cooperation between the earlier and later players. The same holds if we look at the baseline and recommendation treatments separately.
} 
Hence, the recommendation seemed to make the subjects more aware of the intertemporal structure of the game. Nevertheless, the outcome is still far away from a complete cooperative outcome.

The strategies formulated by the subjects help us to understand the failure to arrive at cooperative play. Table 2 summarizes the strategies formulated by the subjects. ${ }^{10}$ In our baseline treatment a large majority of $84.6 \%$ of the subjects develops a strategy that does not make use in any way of the intertemporal structure of the game. Most of these subjects unconditionally choose B. Only 2 subjects spontaneously develop a trigger like strategy.

Table 2

\section{Formulated strategies}

\begin{tabular}{ccc}
\hline & \multicolumn{2}{c}{ treatment } \\
& base-line & recommendation \\
\hline always A & $5(12.8 \%)$ & $2(5.3 \%)$ \\
always B & $28(71.8 \%)$ & $16(42.1 \%)$ \\
recommended strategy & -- & $8(21.1 \%)$ \\
sort trigger & $2(5.1 \%)$ & $12(31.2 \%)$ \\
miscellaneous & $4(10.3 \%)$ & $0(0 \%)$ \\
total & $39(100 \%)$ & $38(100 \%)$ \\
\hline
\end{tabular}

A recommendation to play the grim subgame perfect trigger strategy clearly inspires subjects to develop conditional strategies. ${ }^{11}$ A substantial proportion of the population follows the recommendation. A striking result is that an even larger proportion develops their own trigger strategy. Thus, a slight majority of the subjects (52.3\%) uses a trigger like strategy. With a $x^{2}-$ test the number of trigger versus non-trigger strategies (or constant versus non-constant strategies) is significantly different for the recommendation and baseline treatment (note that

\footnotetext{
${ }^{10}$ A full description of all the strategies is available from the authors.

${ }^{11}$ In the post-experimental questionnaire, 11 of the 38 subjects in the recommendation treatment indicate that the recommendation affected their choice of strategy.
} 
for this test we can use each strategy submitted by a subject as an independent observation). Still, a substantial minority (42.1\%) of the subjects unconditionally chooses defection.

Own creative trigger strategies tend to differ from the recommended strategy in two respects. First, strategies of the 'sort trigger' category tend to be more cautious: 10 of these 14 strategies prescribe to play non-cooperatively if the subject has the second position in the sequence. Second, these strategies tend to be more forgiving: 13 of these 14 strategies do not necessarily select defection if any of the previous players in the sequence selected defection. Usually these strategies resemble tit-for-tat: defect if the previous player chose defection, cooperate if the previous player chose cooperation.

Another difference is rare but remarkable. One strategy in the base line treatment and one strategy in the recommendation treatment are equipped with a variant of the 'defectordetector principle': defect on a defector but do not defect on someone who punishes a defector. Ideally, a strategy with the defector-detector principle cooperates unless an odd number of the direct predecessors defected.

The availability of actual choice data and strategies allows us to investigate the consistency between choices made in the natural time order experiment and the strategies submitted. In the base-line treatment 3 out of 31 choices $(9.7 \%)$ are inconsistent with what would have been prescribed by the strategy submitted. The level of inconsistency is considerably higher in the recommendation treatment. Here, 8 out of 30 choices $(26.7 \%)$ are inconsistent with the strategy. There is a remarkable pattern in the inconsistencies in the recommendation treatment. Five times a subject selects the recommended strategy but plays cooperatively in the natural time order experiment when the strategy would have chosen to defect (the other three inconsistencies arise for other strategies). Probably some subjects only realize the grimness of the recommended strategy when they actually participate in a sequence, and then choose to deviate.

\section{Simulation study of strategies}

Simulations provide insight into how the formulated strategies interact. In the simulations we connect all formulated strategies per treatment. Within a treatment, a computer algorithm randomly selects a strategy and puts it on the second place of the sequence. Then it selects one of the remaining strategies and puts it on the third place. This procedure is repeated until all strategies in a treatment have been assigned a place in the sequence. Then the actual choices produced by this sequence of strategies can be determined. This procedure is repeated 
$1,000,000$ times. The results are summarized in figure 1 . This figure shows the average $\%$ of cooperative choices as function of the place in the sequence.

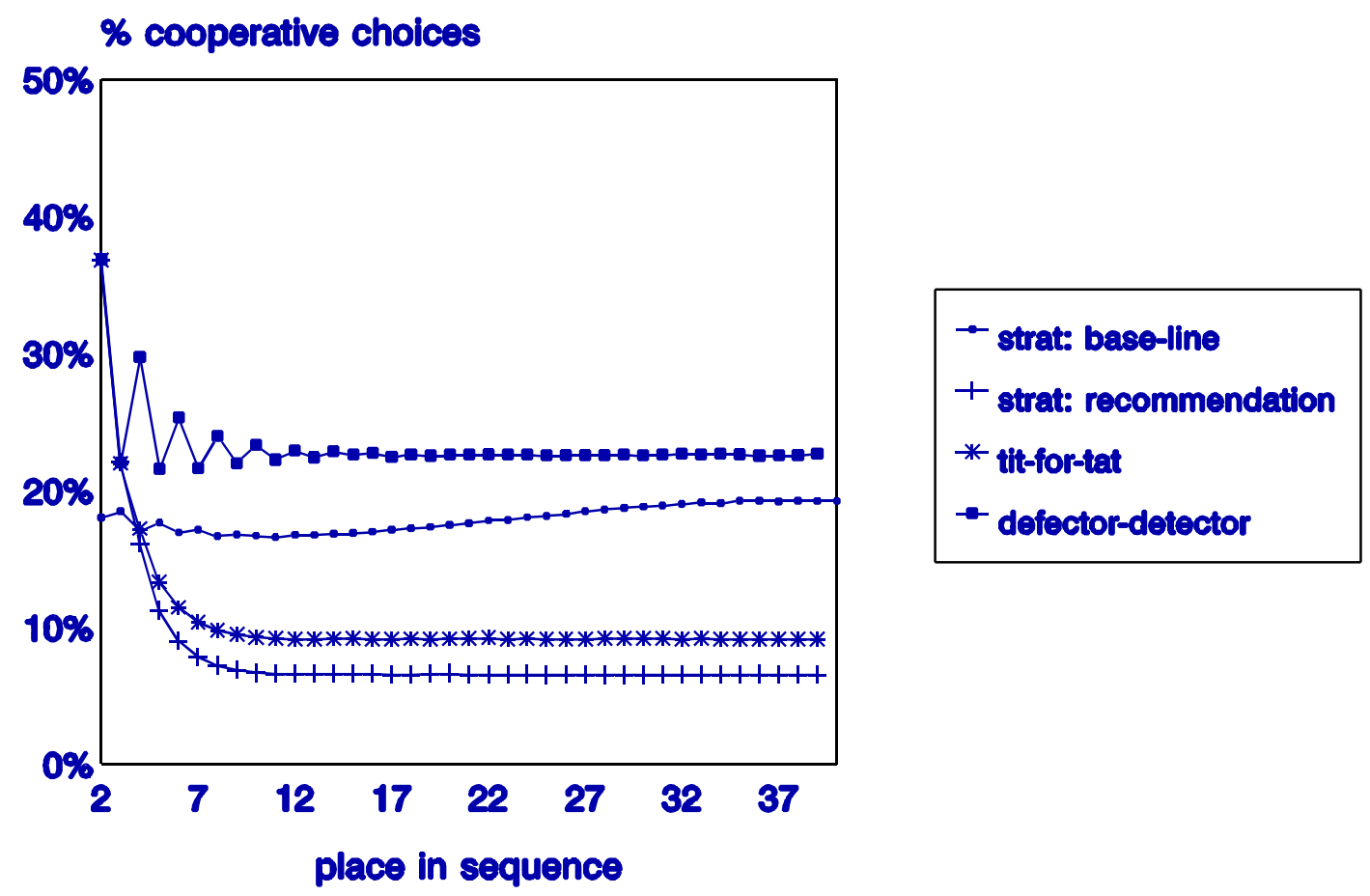

Figure 1: results of simulations of the strategies

The level of cooperative choices in the base-line treatment is more or less constant at $18 \%$ due to the large majority of unconditional strategies. In the recommendation treatment, the level of cooperative choices starts higher at $37 \%$ but drops quickly to $7 \%$, considerably below the level of the base-line treatment. In view of the much lower percentage of unconditional noncooperation in the recommendation treatment this result may seem remarkable at first sight. It is easily explained, however, by the unraveling produced by the combination of the grim trigger strategies and the unconditional noncooperative strategies. Even though in the recommendation treatment more subjects try to exploit the potential for cooperation offered by the intertemporal structure of the game, this only makes matters 'worse'. The large number of unconditional non-cooperative strategies forces the grim strategies to play a large number of non-cooperative choices.

As explained, there are some indications that subjects consider the recommended strategy as being too grim. It could be that the low cooperative level for the recommendation 
treatment is mainly caused by the grimness of the recommended strategy. To investigate this possibility, we carry out simulations for the hypothetical case that the recommended strategy had been 'tit-for-tat' instead of the actual grim strategy, under the (unsubstantiated) assumption that an equal percentage of the subjects would have followed this recommendation. The tit-fortat strategy prescribes to choose cooperatively for the second place and to copy the choice of the predecessor on other places. The other strategies and the relative proportions of the population selecting each strategy are kept constant. Remarkably, as can be seen in Figure 1, the use of the more forgiving tit-for-tat strategy does not substantially alter the results. The level of cooperative choices drops quickly to $9 \%$, still below the level reached without recommendation.

The tit-for-tat strategy does not make an attempt to distinguish between genuine defective choices and defective choices to punish someone who made a genuine defective choice. One rotten apple may affect the whole basket with this strategy. The 'defector-detector' strategy does not have this problem. This strategy prescribes to cooperate unless an odd number of the direct predecessors defected. Simulations replacing the recommended strategy by the defector-detector strategy while keeping other strategies and relative proportions constant show some improvement: now the average level of cooperative choices increases to $23 \%$. Still, even this relatively generous strategy is not able to generate a high level of cooperative choices. The proportion of stubborn defectors in the recommendation treatment $(42.1 \%)$ is simply too high to reach a high level of cooperative choices.

Finally, the simulations also allow us to compare the performance of the various strategies. Table 3 reports the average payoff and the payoff variance for each strategy in each type of simulation. Unsurprisingly, the strategy that unconditionally selects the cooperative choice A generates the lowest average payoff for each of the simulations. Without recommendation the strategy always selecting $\mathrm{B}$ yields a higher payoff than the other strategies. With the actual or a hypothetical recommendation the payoffs of this stubborn noncooperative strategy and the trigger strategies are remarkably close. However, the payoff variance of the stubborn noncooperative strategy is always considerably smaller than the payoff variance of the trigger like strategies.

In sum, the recommended grim trigger strategy has the attractive theoretical property that it sustains cooperation as a subgame perfect equilibrium if it is followed by all players. When not all players follow it, however, it has the unattractive property that players get stuck in noncooperation once one player defects. Our simulations indicate that more forgiving trigger strategies can partially remedy this. In particular, the defector-detector strategy is able to 
substantially increase the aggregate frequency of cooperative play. At the individual level, however, none of the trigger strategies seems able to beat the strategy of unconditional noncooperation. Given the distribution of submitted strategies, the trigger strategies yield about the same expected payoffs but they are much riskier than unconditional noncooperation.

Table 3

Simulated strategies: average payoff and payoff variance

\begin{tabular}{|c|c|c|c|c|}
\hline \multirow[b]{2}{*}{ strategy } & treatment & \multicolumn{3}{|c|}{ recommendation treatment } \\
\hline & $\begin{array}{l}\text { without } \\
\text { recommendation }\end{array}$ & $\begin{array}{l}\text { recommended= } \\
\text { actual grim }\end{array}$ & $\begin{array}{c}\text { recommended= } \\
\text { hypothetical } \\
\text { tit-for-tat }\end{array}$ & $\begin{array}{c}\text { recommended= } \\
\text { hypothetical } \\
\text { defector-detector }\end{array}$ \\
\hline always A & 21.59180 .80 & $22.62 \quad 202.01$ & 29.71291 .88 & $30.05 \quad 293.61$ \\
\hline always B & 37.26229 .90 & 32.5186 .22 & 32.5387 .05 & $35.98 \quad 195.45$ \\
\hline recommended & -- & 32.4594 .45 & -- & -- \\
\hline sort trigger & 34.69250 .31 & 32.13100 .11 & $32.46 \quad 105.42$ & 35.74230 .17 \\
\hline miscellaneous & 30.94249 .31 & -- & -- & -- \\
\hline tit-for-tat & -- & -- & $32.46 \quad 115.38$ & -- \\
\hline defector-detector & -- & -- & -- & $34.43 \quad 319.61$ \\
\hline
\end{tabular}

Note: the first entry denotes the average payoff of the strategy, the second entry (in italics) denotes the payoff variance of the strategy.

\section{Conclusion}

Overlapping generations games have a wide range of application in economics. Many of these applications embody a dilemma between individual rationality and collective efficiency. Theoretical analysis of these games shows that a decentralized solution to this dilemma is possible with infinite overlapping generations. A cooperative outcome can be sustained as a (subgame perfect) Nash equilibrium. This requires players to adopt trigger like strategies. 
This paper provides an experimental investigation of Hammond's Pension Game, the simplest of all overlapping generations games. Our results show that subjects do not easily arrive at a cooperative outcome and do not spontaneously employ trigger like strategies. When we provide subjects with a recommendation to play a subgame perfect grim trigger strategy they seem to become aware of the potential for cooperation offered by the intertemporal structure of the game. A majority of the subjects then forms trigger like strategies, whereas almost nobody does so without a recommendation. However, a substantial part of the population still unconditionally plays non-cooperatively.

Our simulation analysis of the interaction of the strategies reveals that the large fraction of trigger strategies submitted after the recommendation actually makes matters "worse". The presence of both grim trigger strategies and unconditional defectors leads to a very low average level of cooperation. To some degree this is caused by the unforgiving nature of the recommended grim trigger strategy. If these grim triggers were to be replaced by more subtle defector-detector strategies, then in the aggregate the situation would improve. Still, the individual player would be ill-advised to use this defector-detector strategy in our experiment. Given the distribution of submitted strategies, unconditional noncooperation perform equally well in terms of expected payoffs and better in terms of risk.

Theoretically, cooperation can be self-enforcing in overlapping generations games like the one we have implemented. Nevertheless, as our results suggest, cooperation may not be the most likely outcome of such games. We observe little unconditional cooperation and trigger strategies do not seem to come naturally to many people. Therefore, merely establishing that a cooperative outcome is an equilibrium, as is done in many applications of overlapping generations models, is not likely to be a sufficient condition for the occurrence of cooperation. It would seem that other facilitating factors are necessary to actually achieve cooperation. We have investigated one such factor - a general recommendation to play trigger strategies - and found that this alone is not sufficient.

One argument that may be put forward is that people will learn to play cooperatively when they play a game repeatedly. This argument may draw some support from the experimental study by Selten and Stoecker (1986). They find that subjects learn to play a sort of trigger strategy in a repeated game, after they have played many (finitely) repeated games against the same opponent. The experimental study of van der Heijden et al. (1998), however, does not indicate that cooperation rates increase with repeated play of overlapping generations games. Furthermore, for many applications the suggestion that people play overlapping generations games repeatedly does not make much sense. For example, once you have learned 
whether or not your children support you when you are old - possibly in response to how you have treated your parents when you were young - there is no second chance which allows you to revise your strategy. Hence, for many applications of overlapping generations games, any learning should take place during the play of the game, and not across repeated play of the game. One possibility in this direction is that there is direct communication between successive generations of players which may facilitate cooperation (see also Kotlikoff et al., 1988). We leave it to future work to investigate this possibility experimentally.

\section{References}

Abbink, K, and A. Sadrieh (1995), RatImagem - Reasearch Assistance Toolbox for Computer Aided Human Behavior Experiments, Discussion paper No. B-325, University of Bonn.

Bhaskar, V. (1994), Informational Constraints and the Overlapping Generations Model: Folk and Anti-Folk Theorems, mimeo. Delhi School of Economics, presented at the 7th World Congress of the Econometric Society, Tokyo.

Brandts, J., and B. McLeod (1995), Equilibrium Selection in Experimental Games with Recommended Play, Games and Economic Behavior 11, 36-63.

Cadsby, B. and M. Frank, 1990, Experimental Tests of Ricardian Equivalence, Economic Inquiry 29, 645-664.

Cremer, J. (1986), Cooperation in Ongoing Organizations, Quarterly Journal of Economics 101, 33-49.

Hammond, P. (1975), Charity: Altruism or Cooperative Egoism, in: E. Phelps (Ed.), Altruism, Morality, and Economic Theory, New York: Russell Sage Foundation.

John, A., and Pecchenino, R. (1994), An Overlapping Generations Model of Growth and the Environment, Economic Journal 104, 1393-1410.

Kotlikoff, L.J., T. Persson and L.E. Svensson (1988), Social Contracts as Assets: A Possible Solution to the Time-Inconsistency Problem, American Economic Review 78, 662-677.

Ledyard, J. (1995), Public Goods: A Survey of Experimental Research, in: A. Roth and J. Kagel, eds., Handbook of Experimental Economics, Princeton University Press, Princeton.

Lim, S.S., E.C. Prescott and S. Sunder (1994), Stationary Solution to the Overlapping Generations Model of Fiat Money: Experimental Evidence, Empirical Economics 19, 255-77. 
Lucas, R. (1986), Adaptive Behavior and Economic Theory, in: R.M. Hogarth and M.W Reder, eds., Rational Choice: The Contrast Between Economics and Psychology, Chicago and London: University of Chicago Press.

Marimon, R. and S. Sunder (1993), Indeterminacy of Equilibria in a Hyperinflationary World: Experimental Evidence, Econometrica 61, 1073-1107.

Rapoport, A., and Chammah, A. (1965), Prisoner's Dilemma: A Study in Conflict and Cooperation, Ann Arbor: University of Michigan Press.

Salant, D. (1991), A Repeated Game with Finitely Lived Overlapping Generations of Players, Games and Economic Behavior 3, 244-259.

Salant, D., (1995), Behind the Revolving Door: A New View of Public Utility Regulation, Rand Journal of Economics 26, 362-377.

Samuelson, P. (1958), An Exact Consumption Loan Model of Interest with or without the Social Contrivance of Money, Journal of Political Economy 66, 467-482.

Sandler, T. (1982), A Theory of Intergenerational Clubs, Economics Inquiry 20, 191-208.

Selten, R. and R. Stoecker (1986), End Behaviour in Sequences of Finite Prisoner's Dilemma Supergames, Journal of Economic Behaviour and Organization 7, 47-70.

Smith, L. (1992), Folk Theorems in Overlapping Generations Games, Games and Economic Behavior 4, 426-449.

Van der Heijden, E., Nelissen, J., Potters, J., and Verbon, H. (1998), Transfers and the Effect of Monitoring in an Overlapping Generations Experiment, European Economic Review 42, 1363-1391.

Van Huyck, J.B., Gilette, A.B., and R.C. Battalio (1992), Credible Assignments in Coordination Games, Games and Economic Behavior 4, 606-626. 


\section{Appendix. Instructions (translated from Dutch)}

\section{Instructions}

You are about to participate in an experimental study of decision-making. If you follow the instructions of the experiment carefully you may earn a considerable amount of money. All the money you earn will be yours to keep and will be paid to you, privately and confidentially, in cash right after the end of the experiment.

We will distribute the instructions of the experiment now and read them together. After that, you will have the opportunity to ask questions and to practice with the experiment. From now on, you are requested not to talk to, or communicate with, any other participant.

\section{Decisions and earnings}

The participants in the experiments all make one decision when it is their turn. The participants are, so to speak, lined up and every participant in the line will make a decision when all his or her predecessors have made theirs. However, beforehand you will not know when it is your turn to make a decision.

Your decision will affect your own earnings but also the earnings of the subject preceding you in the line (your predecessor). In the same way the choice of the participant after you in the line (your successor) will affect your earnings. The payoff to each participant is thus influenced by her or his own choice and by the successor's choice.

When it is your turn to make a decision, you can select any of two options, A and B. The next table indicates how your choice affects your predecessor's earnings (all amounts run in Dutch guilders).

\begin{tabular}{lll|l|}
\multicolumn{1}{c}{$\begin{array}{c}\text { earnings } \\
\text { predecessor }\end{array}$} & \multicolumn{3}{c}{ your choice } \\
\multirow{2}{*}{$\begin{array}{c}\text { choice } \\
\text { predecessor }\end{array}$} & $\mathrm{A}$ \\
\cline { 2 - 3 } & $\mathrm{B}$ & 50 & $\mathrm{~B}$ \\
\cline { 3 - 4 } & &
\end{tabular}

In the table you can read that if your predecessor opted for A, she or he will earn an amount of 50 guilders if you choose A and she or he will earn an amount of 15 guilders if you choose B. If, however, your predecessor has opted for B, she or he will earn an amount of 70 guilders if you choose for A and she or he will earn an amount of 30 guilders if you choose B.

In the same way as your predecessor's earnings are affected by your choice, will your earnings be affected by the choice of your successor. The table below gives the options.

\begin{tabular}{|c|c|c|c|}
\hline \multirow{2}{*}{\multicolumn{2}{|c|}{$\begin{array}{c}\text { your } \\
\text { earnings }\end{array}$}} & \multicolumn{2}{|c|}{ choice successor } \\
\hline & & A & B \\
\hline your & A & 50 & 15 \\
\hline & B & 70 & 30 \\
\hline
\end{tabular}


In the table you can read that if you opt for A, you will earn an amount of 50 guilders if your successor chooses A and 15 guilders if your successor chooses B. If, however, you would opt for B, you will earn an amount of 70 guilders if your successor chooses A and you will earn 30 guilders if your successor chooses B.

\section{The order of the participants in the line}

As we told you before, you will not know beforehand when it is your turn to make a choice. Everyone has the same probability of being at any particular position in the line. So, there will be a certain, but small probability that you will be the first in the line. This first participant does not make a decision. The earnings of this first participant, however, do depend on the choice of his or her successor, the second participant. If the second participant chooses $\mathrm{A}$, the first participant will receive 50 guilders and if the second participant chooses $\mathrm{B}$, the first participant will receive 30 guilders.

The line will have to end at some point. Therefore, there is a small probability that you are the last participant. The last participant of the line will earn 40 guilders independent of the decision this last participant will make. But the earnings of her or his predecessor will be affected by the choice of the last participant, in the way described above.

In the experiment one of the participants will be selected in a random way and he or she will be given the first position in the line. As noted above, the first participant does not have to make a decision. Thereafter, the second participant will be selected, again in a random way. After this second participant has made a decision, the third participant will be selected, and so on.

If a participant is about to make a decision, there will be a small probability that this participant will be the last in the line. However, if it is your turn to make a decision, you will not know whether you will be the last in the line or not. After a participant has made a choice, a random number generator will determine whether this participant was the last in line, or whether another participant will be added. The probability of being the last participant will always be $10 \%$ and the probability of having another participant added to the line is $90 \%$. This random process was executed by computer before the experiment.

For completeness it should be mentioned that you will never have a second turn in the line. But it might be possible that the line will be closed before it has been your turn to make a decision. In that case, you will receive a fixed amount of 40 guilders for your participation.

\section{Practice}

Before starting the experiment, you will have plenty of time for browsing through the instructions and for practicing the experiment by means of the computer. In doing so, you can practice the procedure and the determination of your earnings. During the practice rounds you are unrelated to any other participant. You have to enter the choices for all participants in the line. The computer will determine the associated earnings. During the practicing, just as in the real experiment, there is a probability of $10 \%$ that a certain participant will be the last participant in the line. In that case the computer will automatically start a new round for practicing. In total, however, no more than 5 practice rounds will be started.

The first practice round will start if you press or mouseclick O. On the screen an announcement is made that the line starts with participant 1 and that you do not have to make a decision. In the upper right part of the screen the earnings of participant 1 are indicated, depending on the successor's choice (participant 2). As the first participant in the line does not have to make a choice, this participant only has to mouseclick OK. So, please click the button OK.

Next participant 2 is the one to make a decision. At the upper right the earnings are indicated dependent on this participant's own choice and the successor's choice. Below that the options A and B 
are indicated. You choose for one of these options by clicking A or B and confirming your choice by clicking OK. Please, enter a choice for participant 2 now.

As soon as you have entered this choice, it will be indicated in the window at the left hand side of the screen. At the same time participant 1's payoff due to participant 2's choice is indicated. The associated payoff for participant 2 cannot be determined until participant 3 (the successor) has made a decision. As soon as this participant has made a decision, the screen on the left will indicate this decision including the associated payoff for participant 2 . After any participant's choice the line will be extended with a new participant with a $90 \%$ probability, and it will be closed with a $10 \%$ probability.

So, you can practice the game by making the decisions for all participants in the line. To be sure, unlike the practice rounds, in the real game to be played shortly you will have to make one decision at most and, moreover, you will participate in one line only. The decisions that you make during the practice rounds will not bear on your payoff during the real experiment.

If you have a question, please raise your hand. One of us will then be at your disposal for answering your question.

\section{[PRACTICE ROUNDS]}

\section{The instructions continued}

In a few minutes you will participate twice in the experiment indicated above. First, you will be asked to formulate a strategy indicating the choice you would like to make contingent on all other possible previous choices in the game. Secondly, you will be asked simply to make a decision when it is your turn. First we will describe the strategy experiment and next we will describe the base experiment. One of these two experiments will determine your payoff. At the end of both experiments, a throw of the die will determine which of the two experiments will be decisive for your earnings. If the die produces 1,2 or 3 , you will get your payoff from the strategy experiment. If the die produces 4,5 or 6 , your payoff will be based on your achievement in the base experiment.

\section{Strategy experiment}

In this experiment we are asking you to do more than just choosing between $\mathrm{A}$ and $\mathrm{B}$. We would like you to formulate a strategy for this experiment. A strategy is a general rule indicating your actual choice contingent on all cases that may occur in the game.

Formulating a strategy would be easy if you were to be sure that you would be the second participant in the line. In that case you would know that the first participant would not make a choice. Being the second participant you only had to choose between A and B. Also if you were sure that you would be the third participant in the line, a strategy would be a relatively simple affair. In that case you would know that the first participant in the line would not make a choice and that the second participant in the line would opt for either A or B. A strategy for participant 3 then indicates what you would choose in one of the two cases: "If the second participant chooses A, I will opt for ....(A or B), and if the second participant chooses B, I will opt for ....(A or B)" This is an example of a strategy because in all possible contingencies it indicates your decision. For the fourth participant a strategy can be somewhat more complicated, because the decision you would like to make as participant 4 can be dependent on participant 3's choice as well as on participant 2's choice.

In this experiment formulating a strategy is even more complicated as you do not know what position in the line you will have. This means that you will have to formulate your strategy in a more general way. Of course, it is up to you how complicated your strategy is going to look like. The only criterium your strategy has to satisfy is that it has to be complete, i.e. for all possible cases that can be encountered your strategy has to pick a choice. So, a strategy is an action plan indicating your choice (A or B), where these choices might be dependent on the decision(s) made by one or more previous 
successors. Of course, your strategy cannot be dependent on decisions by participants who are after you in the line.

After having formulated your strategy, we will use your strategy for determining your choice, just as we will use other participants' strategies to determine their choices. The procedure described earlier will determine your position in the line. Dependent on your predecessors' choices, your strategy will fix your choice. Your choice and your successor's choice will determine your payoff (if the die throw indicates that the strategy experiment is decisive for your payoff).

To help you in formulating a strategy in the attached strategy form we classified the possible strategies in different categories. Strategies of Category 0 are the most simple. A strategy in this category implies that you will always decide for A or B independent of your position in the line and independent of all your predecessors' choices. A strategy of Category 1 is somewhat more complicated as it indicates that your choice will depend on your predecessor's choice. A strategy of Category 2 admits your choice to depend on the choice of your predecessor as well as on the choice of the predecessor of your predecessor. If you opt for a strategy of Category 3, your choice can be dependent on the choice of three participants preceding you in the line. Finally, Category 4 is a "remainder category" containing those strategies that cannot be classified into Categories 0-3.

First you have to determine the Category your strategy fits in; next you have to fill in your strategy in the appropriate place on the strategy form. You will have ample time for determining your strategy and for asking questions about the formulation of your strategy or about the procedure. If you are finished formulating your strategy you are requested to raise your hand. We will take in your strategy after having checked it for completeness.

Recommendation (only in the recommendation treatment)

One of the participants in a previous experiments made a recommendation to the participants in future experiments which amounted to the following:

"I recommend that the second participant chooses A. I recommend that each subsequent participant chooses A if all predecessors have chosen A and chooses B in all other cases. If everyone follows this recommendation, it is beneficial for every participant to choose A. A choice for A will be 'rewarded' with a choice for A by the successor, whereas a choice for B will be 'punished' with a choice for B by the successor. In this manner, the payoff to all participants will be highest."

If you want to follow this strategy, you can indicate this by entering "recommended strategy" on your strategy form.

[Subjects formulate strategies.]

[Forms of the five strategy classes are available from the authors.]

\section{Base experiment}

Before playing out the strategy experiment, we will first conduct the experiment once as described earlier. This time there is no need for you to formulate a strategy, but you simply choose A or B if it is your turn. You will be selected one after the other for making a choice.

Each participant will see on the screen when it is her or his turn to make a choice. 